\title{
Concrete Stress-Strain Characterization by Digital Image Correlation
}

\author{
Saldaña HA, ${ }^{1}$, Márquez Aguilar $\mathrm{PA}^{2 *}$ and Molina $\mathrm{OA}^{2}$ \\ ${ }^{1}$ School of Chemical Sciences and Engineering is also from the Autonomous University of the State of Morelos \\ ${ }^{2}$ Research Center for Engineering and Autonomous University of the State of Morelos Av. University \# 1001, Col. Chamilpa. Cp. 62209. Cuernavaca Morelos, Mexico
}

\begin{abstract}
In mechanics of materials it is important to know the stress-strain relation of each material in order to understand their behaviour under different loads. Concrete is one of the most used materials in structural mechanics and they are always under axial loads. This work is implemented using one beam and the speckles created by its reflection. Strain field measurement with noninvasive techniques is needed in order to sense rough-like materials. We present an experimental approach that describes the mechanical behavior of structural materials under compression tests, which are done in a universal testing machine. In this work we show an evaluation of the in-field measurements obtained by digital image correlation allowing us to evaluate the heterogeneous strain evolution observed during these test.
\end{abstract}

\section{Introduction}

Stress-strain diagrams are very important in understanding the behaviour of materials under load [1]; they show the elastic, plastic and rupture part of materials. There are two methods to obtain the diagram: the invasive methods in which mechanics take advantage doing physical tests as the test-tube in which is placed a small piece of the probe and it is applied a specific load to it, then is measured the deformation in displacement [2]. Optical methods are also used as an invasive way to determine residual stress, in-field displacements and strain, in which the most used technique, is the hole-drilling method, created in 1930 by Mathar [3], nowadays this technique is standardized by ASTM [4]. When a laser light felt on a matt surface such as paper or rubber-like material, a speckle pattern is formed and a high-contrast grainy pattern will be reflected. This effect was called egranularity by Rigden and Gordon [5]. The simplest image matching procedure is cross-correlation (CC) that can be performed either in physical space [6,7]; in Fourier space [8] by using Fast Fourier Transforms (FFT) one can evaluate the CC function very quickly. Anuta [9] took advantage of the high speed of FFT algorithm doing digital multispectral and multi-temporal statistical pattern-recognition. Kuglin and Hines [10] observed that information about the displacement of one image with respect to another is included in the phase component of the crosspower spectrum of the images. Correlation technique was applied analysing the strain evolution of a rubber-like material under multiaxial stresses [11]; also for strain measurements using a Vic 2D system in order to obtain the strain diagram of a compound material [12] and it is also applied in resistance heating tensile test [13].

Concrete is one of the most used materials in structural mechanics, in 1988 Rots and Borst loaded a piece of concrete in a tension test and they got around $3 \mathrm{~N} / \mathrm{mm}^{2}$ and $0.02 \%$ stress-strain respectively [14], this material was used for the development of techniques in modelling the seismic response [15]; Wasantha found a relation between stress-strain for concrete under heavy vehicle loadings [16].

The development of non-destructive testing methods is the main challenge for the assessment of structural elements in existing constructions. In this work we measure in-plane strain of a common structural material subjected to a continuum axial load. A compression test is done to analyse the strain evolution of our sample and Digital Image Correlation (DIC) combined with CC method are done in order to obtain in-plane strain deformation, in which we use one laser beam impinging our material in its cross-section during the compression test.
From these experimental tests, we correlate the stress-strain diagram with in-field strain measurements, also it is included the accuracy and mean errors of the tests.

\section{Developments}

\section{Speckles statistics}

The random intensity distribution is called speckle pattern, which is formed when coherent light is reflected from a rough surface or when light is propagated through a medium with random refractive index fluctuations [17]. In general the statistical properties of speckle patterns depend both on the coherence of the incident light and the detailed properties of the random surface or medium.

The surfaces of most materials are extremely rough on the scale of an optical wavelength ( $\lambda \approx 5 \times 10^{-7}$ meters). When monochromatic light is reflected from such a surface, the optical wave resulting at any distant point consists of many coherent components; the interference of all the components is then called the speckle pattern.

Let a speckle signal be $u(x, y, z ; t)$ at an observation point $(x, y, z)$ and time $t$ with $u$ as a fully monochromatic wave, the analytic signal takes the form:

$$
u(x, y, z ; t)=A(x, y, z) \exp ^{(i 2 \pi v t)}
$$

where $v$ is the optical frequency and $A$ represents the phasor amplitude of the field, which is a function of space [18]. When a speckle pattern arises by free-space propagation, the amplitude of the electric field at a given observation point $(x, y)$ consist in every de-phased contributions from all different scattering regions of the rough surface, then the

*Corresponding author: Márquez Aguilar PA, Research Center for Engineering and Applied Science University of the State of Morelos Av. University \# 1001, Col. Chamilpa. Cp. 62209. Cuernavaca Morelos, Mexico, Tel: (52 777) 3297000 E-mail: pmarquez@uaem.mx

Received December 12, 2015; Accepted December 24, 2015; Published December 26, 2015

Citation: Saldaña HA, Márquez Aguilar PA, Molina OA (2015) Concrete StressStrain Characterization by Digital Image Correlation. J Appl Mech Eng 4: 189. doi:10.4172/2168-9873.1000189

Copyright: (c) 2015 Saldaña HA, et al. This is an open-access article distributed under the terms of the Creative Commons Attribution License, which permits unrestricted use, distribution, and reproduction in any medium, provided the original author and source are credited. 
amplitude $A(x, y, z)$ is represented as a sum of every contribution $k=1,2 \ldots \ldots, N$ :

$$
A(x, y, z)=\sum_{k=1}^{\infty} \frac{1}{\sqrt{N}} a_{k}(x, y, z)=\sum_{k=1}^{\infty} \frac{1}{\sqrt{N}} a_{k} \exp ^{\left(i \varphi_{k}\right)}
$$

Equation (2) must follow some statistical properties [17,18]: The amplitude $a_{k} / \sqrt{ } N$ and the phase $\varphi_{k}$ of each element are statistically independent of each other. The phases are uniformly distributed between $(\pi,-\pi)$.

In order to study the intensity distribution of the resultant field is necessary to take the real and imaginary part of Eq. (1):

$$
\begin{aligned}
& A^{(r)}=\frac{1}{\sqrt{N}} \sum_{k=1}^{\infty}\left|a_{k}\right| \cos \varphi_{k} \\
& A^{(i)}=\frac{1}{\sqrt{N}} \sum_{k=1}^{\infty}\left|a_{k}\right| \sin \varphi_{k}
\end{aligned}
$$

When assumptions of the statistical properties are considered it is possible to stablish:

$$
\begin{aligned}
& {\left[A^{(r)}\right]^{2}=\frac{1}{N} \sum_{k=1}^{\infty} \sum_{m=1}^{\infty}\left|a_{k} \| a_{m}\right|\left(\cos \varphi_{k} \cos \varphi_{m}=\frac{1}{N} \sum_{k=1}^{\infty} \frac{\left[\left|a_{k}\right|\right]^{2}}{2}\right.} \\
& {\left[A^{(i)}\right]^{2}=\frac{1}{N} \sum_{k=1}^{\infty} \sum_{m=1}^{\infty}\left|a_{k} \| a_{m}\right|\left(\sin \varphi_{k} \sin \varphi_{m}=\frac{1}{N} \sum_{k=1}^{\infty} \frac{\left[\left|a_{k}\right|\right]^{2}}{2}\right.}
\end{aligned}
$$

thus is seen that real and imaginary parts of complex field have identical variances and are uncorrelated. Generally $N$ is a number extremely large so real and imaginary parts can be expressed by $(3,4)$ as sums of a very large number of independent random variables. It follows from the central limit theorem [17] that as $N \rightarrow \infty, A^{r}$ and $A^{i}$ are asymptotically Gaussian. Coupling this fact with the results of $(5,6)$, the joint probability density function of the real and imaginary parts of the field is:

$$
P_{r, i}\left(A^{(i)}, A^{(i)}\right)=\frac{1}{2 \pi \sigma^{2}} \exp \left[\frac{\left(\left[A^{r}\right]^{2}+\left[A^{i}\right]^{2}\right)}{2 \sigma^{2}}\right]
$$

where

$$
\sigma^{2}=\frac{1}{N} \sum_{k=1}^{\infty} \frac{\left[\left|a_{k}\right|\right]^{2}}{2}
$$

This kind of density function is commonly known as a circular Gaussian density function. One of the most important parameters measured in optics is the intensity, thus according from the known statistics of the amplitude is necessary to find the corresponding statistical properties of the intensity. Therefore the intensity $I$ and the phase $\theta$ of the field are related to the real and imaginary parts of the amplitude [19]:

$$
\begin{aligned}
& A^{(r)}=\sqrt{I} \cos \theta \\
& A^{(i)}=\sqrt{I} \sin \theta
\end{aligned}
$$

Substituting equations (9) and (10) in (7) we can observe that the probability distribution of intensity would be:

$$
P_{i, \theta}(I, \theta)=\frac{1}{4 \pi \sigma^{2}} \exp \left[\frac{-I}{2 \sigma^{2}}\right]
$$

\section{Image correlation}

Digital image correlation is an optical method that uses a mathematical correlation analysis to examine digital image data taken while samples are in mechanical tests. This technique consists on capture consecutive images with a digital camera during the deformation period in order to evaluate the change in surface characteristics and understand the behavior of the specimen while it is subjected to an increasing load.
It is well known that for cross correlation technique sequenced images are needed. We consider a plane covered by a speckle pattern as the image intensity at time $t_{0}$ defined by $f(x, y)$, where the two dimensional FFT is:

$$
F[f(x, y)]=F(u, v)=\iint_{-\infty}^{\infty} f(x, y) \exp ^{-i 2 \pi(u x+v y)} d x d y
$$

A second image is considered where the intensity is in a time $t_{1}=t_{0}+\Delta t$ defined by $g(x, y)$; assuming $g(x, y)$ as a translation of $f(x, y)$ we have: $g(x, y)=f\left(x-x_{0}, y-y_{0}\right)$. Cross-spectrum $I(u, v)$ can be defined by the multiplication of two signals, considering $F$ the Fourier transform of the first and $G$ the Fourier transform of the second and ${ }^{\star}$ the FFT complex conjugate we get:

$$
I(u, v)=F(u, v) \bullet G^{*}(u, v)=F(u, v) \bullet F(u, v) \exp ^{i 2 \pi\left(u x_{0}+v y_{0}\right)}
$$

in Eq. 13 it can be seen that the displacement can be expressed according to two components corresponding to two directions; therefore the phase can be decomposed into two expressions with a variation between $-\pi$ and $+\pi$ [13].

In order to get cross correlation we need to take the inverse Fourier transform of the cross spectrum. Lets call $U$ and $V$ a pair of 2-D images, where $U$ represents the reference image and $V$ the deformed image, it is taken the FFT form both images and cross-correlation is defined by:

$$
C C=F^{-1}\left[F(U) \bullet F^{*}(V)\right]
$$

where $F$ implies 2-D Fourier transform, $F^{-1}$ implies 2-D inverse Fourier transform. The use of FFT requires that images $U$ and $V$ are the same size and have dimensions that are powers of 2 . In the present work, we analyze $2^{10} x 2^{10}$ images in order to map a bigger area of the whole image and the shift $\delta x(=\delta y)$ between two consecutive images is 128 pixels in order to get a better result. These two parameters define the mesh formed by the images used to describe the displacement field [11].

\section{Compresion tests}

Physical tests are used in order to know mechanical properties of materials and compression test is one of these tests which enable the user to understand the behavior of a material under a continuous axial load; from this test we obtain the stress-strain diagram [2]. In this work we made a mixture of sand-cement with a ratio of $3 \times 1$ respectively with dimensions of $5 \times 5 \times 4.5 \mathrm{~cm}$. and they undergo into compression test according to ASTM E-9 [20]. The tests were performed with a speed ratio of $0.5 \mathrm{~mm} / \mathrm{s}$ up to $3 \mathrm{GPa}$ approx. Since we are working on the elastic part of the diagram, therefore we can apply Hooke's law:

$$
\sigma=E \bullet \varepsilon
$$

where $\sigma$ is the stress, $E$ is the Young modulus of the material: 2.2 GPa experimentally obtained by the universal machine and $\varepsilon$ is the dimensionless deformation.

In DIC procedure, when the same reference picture is used, it is not possible to measure large displacements in a sequence of pictures, but when they remain small enough it is possible to assume the first image as the reference for the whole analysis. In the present work, we work with deformations less than two millimeters. It is well-known that the infinitesimal strain tensor $\varepsilon_{c}$ is well adapted to small displacements and it can be evaluated as:

$$
\varepsilon_{c}=\frac{1}{2}\left(R+R^{T}\right)-1
$$

where $\varepsilon_{c}$ is the strain, $R$ is an orthogonal second rank tensor and $R^{T}$ implies transpose. 


\section{Theoretical analysis}

As it is well-known, laser beams propagates a unidirectional wave with some divergence and with a finite cross-section; the most common output for such a beam is Gaussian in the TEM ${ }^{00}$ mode, which its intensity distribution is:

$$
I(r)=I_{0} \exp \left[\frac{-2 r^{2}}{w^{2}}\right]
$$

where $r=\left(x^{2}+y^{2}\right)^{1 / 2}$ and $w$ is the spot size and depends on the $\mathrm{z}$-coordinate [21]. The intensity profile is a Gaussian and it maintains its profile while it propagates. The Gaussian beam equation is deduced from Helmholtz equation and is represented by [22]:

$$
E(r, z)=A \frac{w_{0}}{w(z)} \exp \left[\frac{-r^{2}}{[w(z)]^{2}}+\frac{k r^{2}}{2 R(z)}+k z-\eta(z)\right]
$$

where $w_{0}$ is the beam waist, $w(z)$ is the beam spot size, $R(z)$ is the curvature radius of the spherical waves and $\eta(z)$ is the beam phase angle [23]. And the intensity distribution of a Gaussian Beam is:

$$
I(r, z)=I_{0} \frac{w_{0}^{2}}{[w(z)]^{2}} \exp \left[\frac{-2 r^{2}}{[w(z)]^{2}}\right]
$$

Gaussian beams are able to pass through different media; the light reflection occurs when it arrives to the boundary separating two media of different optical densities and some of the energy is reflected back into the first medium [24], taking this outset, our laser-beam strikes a rough material and the reflection can be studied as a speckle pattern. The ratio between the intensity of the reflected beam and the incident beam is called reflectivity and is expressed by:

$$
R=\frac{I_{r}}{I_{i}}
$$

where $I_{r}$ and $I_{i}$ are the reflected and incident beams respectively and when a beam pass through the media, there exist transmissivity and according to the conservation law of energy [25]:

$T+R=1$

In the present work, as we are working with solid materials, the transmissivity is zero. Therefore, we can assume that:

$$
I_{i} \bullet R=I_{r}
$$

for $\mathrm{R} \leq 1$ and according to cross-correlation analysis Eq. (14), taking $U$ and $V$ as speckle patterns and using Eq. (11), expanding formally and changing coordinates we obtain

$$
\begin{aligned}
& F(u, v)=\iint_{-\infty}^{\infty} \frac{1}{4 \pi \sigma^{2}} \exp \left[\frac{-\left(x^{2}+y^{2}\right)}{2 \sigma^{2}}\right] \exp ^{-i 2 \pi(u x+v y)} d x d y \\
& U(\rho: t)=\frac{1}{4 \sigma^{2}} \exp \left[\frac{\pi^{2} \sigma^{2}}{2} \rho^{2}\right]
\end{aligned}
$$

Being $U(\rho: t)$ the first image Fourier transform at an initial time $t_{0}$; the same process is done for $V(\rho: \Delta t)$, where $V$ is the second image Fourier transform at a time $t_{1}$ and cross correlation is defined by:

$$
C C=\left[\frac{1}{4 \sigma^{2}}\right]^{2} \exp ^{i 2 \pi(\Delta x+\Delta y)}
$$

Finally it is taken the correlation phase from the exponential and infinitesimal strain tensor takes place obtaining in-field measurements. From Eq. (25) is possible to see that the relation will keep a Gaussian form, taking this outset, it is assumed that this problem is similar to a common reflection, where the scattered area will decrease and such decrement will be proportional to the deformation.

\section{Experimental Setup and Data Processing}

Three probes were made by adding $3 \times 1$ sand-cement mixture and they were placed in the universal testing machine in order to begin the compression tests. A diagram of this method is shown schematically in Figure 1a. The output of a Diode-Pumped Solid-State laser (L) with a wavelength $\lambda=532 \mathrm{~nm}$ and power of $200 \mathrm{~mW}$ [26], is propagated through a positive lens (l) which is placed in front of the sample (Sa) in order to irradiate the cross-section face and the scattering reflection impacts a screen (Sc) which is placed aside the laser beam; the material is first completely flat and it is compressed by the machine (M). During the compression tests, the speed of the compression load was $0.5 \mathrm{~mm} / \mathrm{s}$ with duration of 20 minutes approximately and while they were taking place the speckle reflection was recorded with a high resolution video camera (Vc) (Figure 1b).
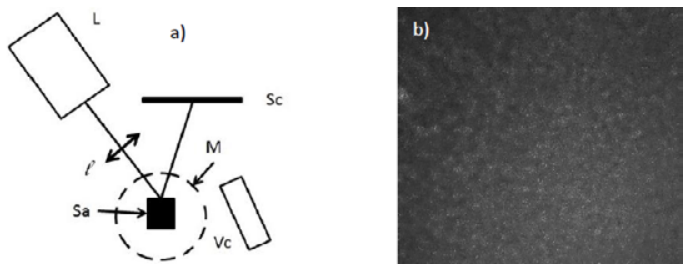

Figure 1: Experimental set-up: (a) material with no strain; (b) speckle photogram.

Once the video is recorded, it is divided into thousands of photograms in order to load each image and process it. A program is written in Matlab for Digital Image Correlation (DIC). The crosscorrelation calculation is described below: in Figure 2a it is shown how the code loads the image and it is converted into a gray-scale image as it is shown in Figure 2b; the image is crop into a $1024 \times 1024$ in order to begin the FFT analysis of all the images, taken the first as the reference; from the second image and forward, they are considered as deformed images.
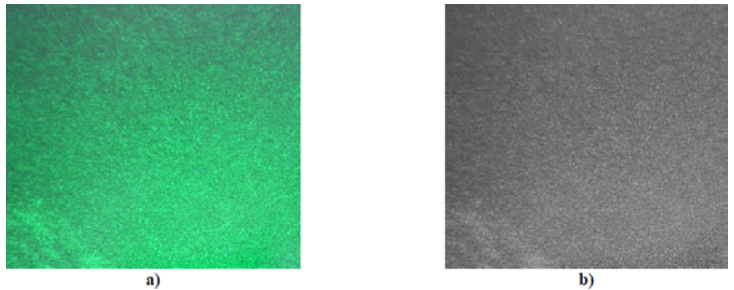

Figure 2: Cross correlation technique, a) loaded image; b) grey-scale image.

Once it is done the cross correlation technique we obtain the phase component as Kuglin and Hines show [10]. Therefore we apply a shift between each interval $\delta \times=\delta \mathrm{y}=128$ pixels as is shown in Figure 3 .

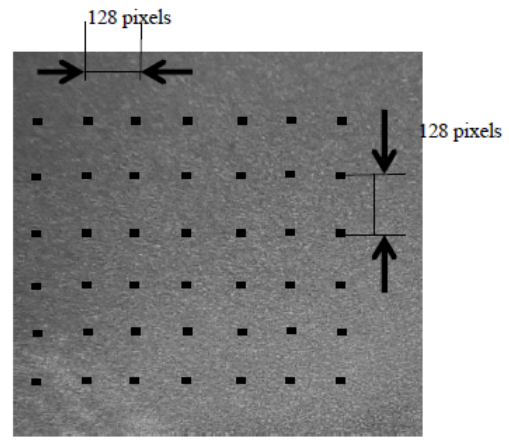

Figure 3: Displacement contours $\delta x=\delta y=128$ pixels 
The first strain measured by the universal machine is 0.002 while cross correlation technique allows us to measure 0.0019349; the shear component is equal to or less than $6.1 \times 10^{-5}$. It has been reported that for pure rotation measurements, this technique had $2 \times 10^{-4}$ sensitivity [13].

\section{Results and Discussion}

In the section above, we mentioned that three samples were prepared for compression test, for each one has been obtained two graphs of interest, the strain diagram is shown in Figure 4 and the DIC plot which is shown in Figure 5, both graphs correspond to the first sample.

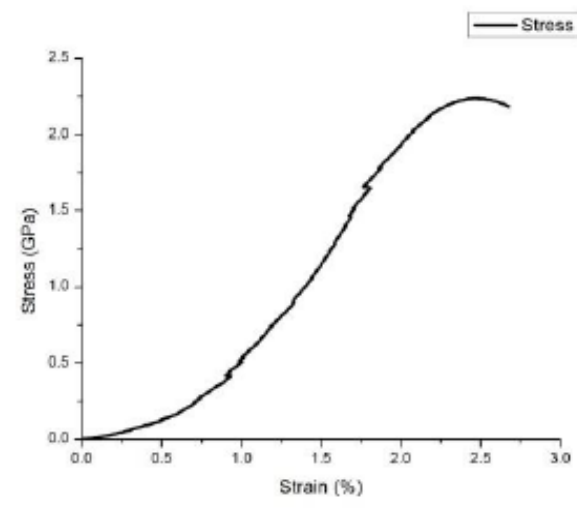

Figure 4: Real stress-strain diagram.

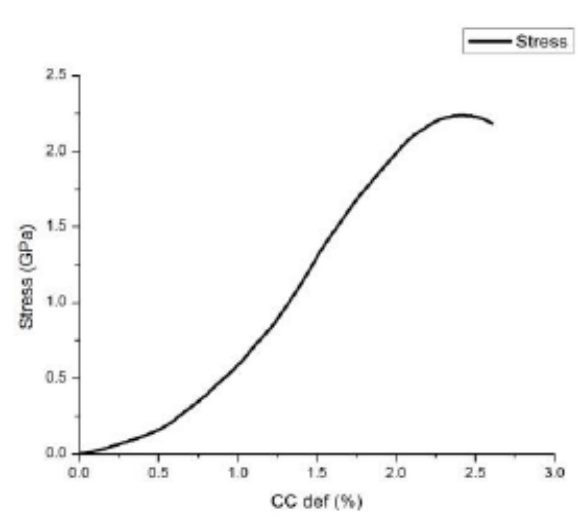

Figure 5: Experimental stress-strain diagram.

In Figure 4 we plot the strain diagram obtained from the machine and in Figure 5 we plot the stress-strain diagram from the experimental results obtained by Eq. (16). For both graphs we see a linear behavior.

In the next figure we compare the real deformation and the experimental data obtained, also some statistical results are shown in Table 1.

In Figure 6 we plot both graphs of interest, stress-strain from the machine and cross-correlation deformation from Eq. (15) (black) and from Eq. (16) (red) respectively. These results are shown for each sample; in Table 1 it is shown the statiscis of the first test, it was observed that there are such a good correlation obtaining $6.4 \%$ error from the real strain measurement.

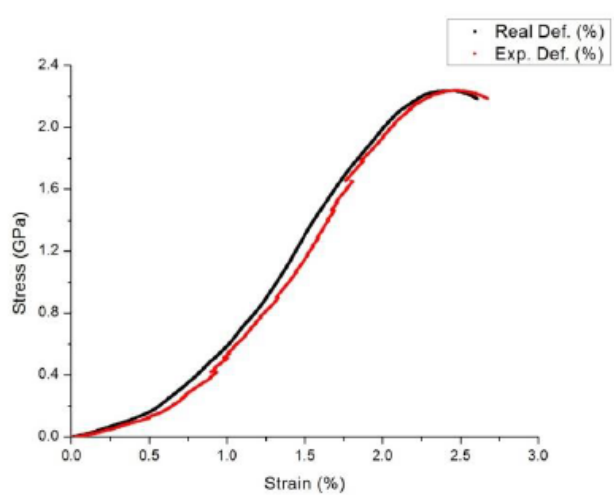

Figure 6: Comparison between stress-strain diagrams for sample 1.

\begin{tabular}{|l|c|}
\hline \multicolumn{2}{|c|}{ 1st $^{\text {st }}$ Sample } \\
\hline Difference from real $\mathrm{E}$ & $2.17 \pm 0.8 \mathrm{Gpa}$ \\
\hline Mean relative error $\varepsilon$ & $6.40 \%$ \\
\hline Standard deviation & 1.45 \\
\hline
\end{tabular}

Table 1: Statistical results for sample 1 during the compression tests and crosscorrelation analysis.

In Figure 7 it is plot both graphs of interest and Table 2 summarises showing the statiscis of this sample, it was observed a better correlation than sample 1 obtaining $5 \%$ error from the real strain measurement.

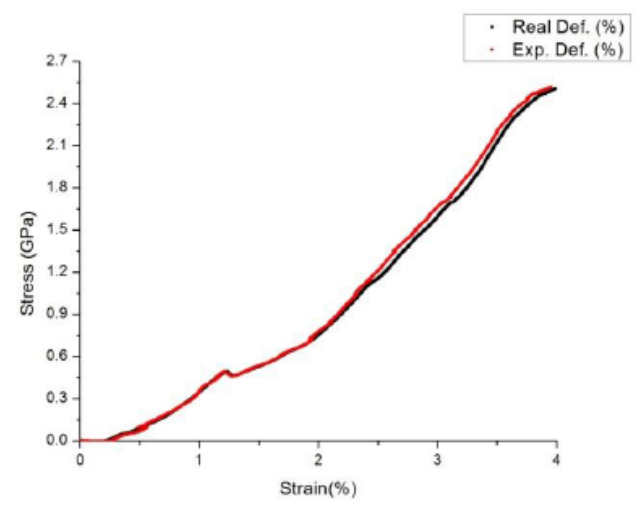

Figure 7: Comparison between stress-strain diagrams for sample 2.

\begin{tabular}{|l|c|}
\hline \multicolumn{2}{|c|}{$2^{\text {nd }}$ Sample } \\
\hline Difference from real E & $2.19 \pm 0.5 \mathrm{Gpa}$ \\
\hline Mean relative error $\varepsilon$ & $5.00 \%$ \\
\hline Standard deviation & 1.9 \\
\hline
\end{tabular}

Table 2: Statistical results for sample 2 during the compression tests and crosscorrelation analysis

In Figure 8 it is plot both graphs of interest and Table 3 summarises showing the statiscis of this sample, it was observed a simmilar behaviour than sample 1 obtaining $6 \%$ error from the real strain measurement. 


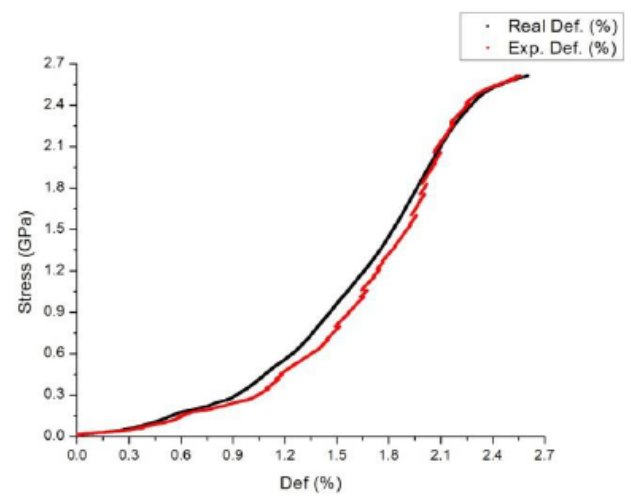

Figure 8: Comparison between stress-strain diagrams for sample 3.

\begin{tabular}{|l|c|}
\hline \multicolumn{2}{|c|}{$3^{\text {rd }}$ Sample } \\
\hline Difference from real E & $2.17 \pm 0.8 \mathrm{Gpa}$ \\
\hline Mean relative error $\varepsilon$ & $6.10 \%$ \\
\hline Standard deviation & 1.45 \\
\hline
\end{tabular}

Table 3: Statistical results for sample 3 during the compression tests and crosscorrelation analysis.

For samples 1 and 3 the behaviour of the experimental graph is below of the real stress-strain diagram but for sample 2 it is more correlated and behaves upper than real measurement. As they are all samples made by the same mixture, all of them behave approximately the same but the speckle reflection differs because of their surface, that's why we have got these results.

In Table 4 we show the accuracy and mean error of each measurement, so we can assume that the mean accuracy of our method is $94.4 \%$.

\begin{tabular}{|c|c|c|}
\hline Probe & Accuracy & Error \\
\hline 1 & $94 \%$ & $6 \%$ \\
\hline 2 & $95 \%$ & $5 \%$ \\
\hline 3 & $94 \%$ & $6 \%$ \\
\hline
\end{tabular}

Table 4: Accuracy and error of the test.

By comparing the average strain determined for three different specimens with the compression stress values measured with the load cell, it was demonstrated that the present technique can measure the relative strain with an average uncertainty of $5.6 \%$.

\section{Conclusion}

It was measured the strain of a rough-like material under a compression test using one laser beam, it's speckle reflection and digital image correlation treatment, obtaining $94.4 \%$ accuracy. We obtain $6.1 \times 10^{-5}$ sensitivity, which is less than the reported $2 \times 10^{-4}$. We demonstrate that cross-correlation plus speckle metrology could be a reliable technique for measuring strain such as standardized compression tests.

\section{Acknowledgement}

Alonso Saldaña Heredia wants to thank CONACYT for the grant No. 360140 .

\section{References}

1. Beasley $F$ (2011) Theory and Design for Mechanical Measurements. $3^{\text {rd }}$ edn USA John Wiley and Sons.
2. Gere J, Goodno B (2009) Mecánica de Materiales $7^{\text {th }}$ edn. México Cengage Learning.

3. Mathar J (1934) Determination of Initial Stresses by Measuring the Deformation Around Drilled Holes. Transactions ASME 56: 249-254.

4. (2008) ASTM Determining Residual Stresses by the Hole-Drilling Strain Gage Method. ASTM Standard Test Method E837-08 American Society for Testing and Materials West Conshohocken PA.

5. Rigden JD, Gordon El (1962) The granularity of scattered optical laser light Proceedings of the Institute of Radio Engineers 50: 2367-2368.

6. Peters WH, Ranson WF(1982) Digital image techniques in experimental stress analysis. Opt Eng 21: 427-441.

7. Sutton MA, Mc Neill SR, Helm JP, Chao YJ (2000) Advances in two dimensional and three dimensional computer vision. Photomechanics Topics in Applied Physics 77 Springer Berlin. 323-372.

8. Chen DJ, Chiang FP, Tan YS, Don HS (1993) Digital speckle-displacement measurement using a complex spectrum method. Appl Opt 32:1839-1852.

9. Anuta PE (1970) Spatial registration of multispectral and multitemporal digital imagery using fast Fourier transform techniques. IEEE Trans Geosci Electron 8: 353-368.

10. Kuglin CD, Hines DC (1975) The phase correlation image alignment method. In Proc. Int'I Conf Cybernetics and Society: 163-165.

11. Chevalier L, Calloch S, Hild F, Marco Y (2001) Digital image correlation used to analyze the multiaxial behavior of rubber-like materials. Eur $\mathrm{J}$ mech $\mathrm{A} /$ solids 20: 169-187

12. Cintrón R, Saouma V (2008) Strain measurements with digital image correlation system Vic-2D. The George E Brown Jr. Network for Earthquake Engineering Simulation CU-NEES-08-06.

13. Pradille C, Bellet M, Chastel Y (2010) A Laser speckle method for measuring displacement field Application to resistance heating tensile test on steel. Applied Mechanics and Materials 24: 135-140.

14. Rots J, Borst R (1989) Analysis of concrete fracture in "direct" tension. Int J Solids Structures 25: 1381-1394.

15. Gerin M, Adebar P (2004) Accounting for shear in seismic analysis of concrete structures. $13^{\text {th }}$ World Conference on Earthquake Engineering Vancouver B.C, Canada Paper No. 1747.

16. Wasantha MA (2005) Analysis and verification of stresses and strains and their relationship to failure in concrete pavements under heavy vehicle simulator loading. Ph.D. Thesis University of Florida USA.

17. Dainty JC (1975) Laser speckle and related phenomena. Springer-Verlag Berlin Heidelberg 9.

18. Goodman JW (1976) Some fundamental properties of speckle J Opt Soc Am 66: $1145-1150$.

19. Fontenelle H (2009) Laser speckle imaging: spatio-temporal image enhancement. Ph.D Thesis University of Patras Greece.

20. ASTM E-9 ICS Number Code 77.040.10 (Mechanical testing of metals).

21. Sirohi RS (2009) Optical methods of measurement. Wholefield techniques $2^{\text {nd }}$ Edition Taylor and Francis Group. USA.

22. Alda J (2003) Laser and Gaussian Beam Propagation and transformation Encyclopaedia of optical engineering.

23. Yariv A (1990) Quantum Electronics $3^{\text {rd }}$ edn. USA John Wiley \& Sons.

24. Wood R (1988) Physical Optics $3^{\text {rd }}$ edn. Washington DC Optical Science of America.

25. Born M, Wolf E (1970) Principle of optics $4^{\text {th }}$ ed. Pergamon Press UK

26. (2015) Laserglow Technologies LCS-0532 Low-Cost DPSS Laser System Laserglow Part Number: C53200XSX Laser Product Datasheet generated. 